Method 1058 workers at a government office in Nigeria were enrolled in a study of body size, dietary energy intake, physical activity and anthropometric characteristics.

Results The mean age (SD) of participants was 41.5 (9.3) years and mean BMI (SD) was 27.0 (4.9), mean waist (SD) $=75.4(26.8) \mathrm{cm}$, mean hip circumference $(\mathrm{SD})=86.7(33.1) \mathrm{cm}$, mean waist hip ratio $(\mathrm{WHR})=0.88(0.10)$ and mean weight $(\mathrm{SD})=74.6(14.2) \mathrm{kg}$. There was strong correlation between the interviewee perception of their current body type and that of the interviewer $(r=0.73, p<0.001)$. Compared with anthropometric characteristics, the body images correlated most strongly with BMI $(\mathrm{r}=0.56, \mathrm{p}<0.001)$ but less so with waist circumference $(r=0.33)$, hip circumference $(r=0.33)$, WHR ( $r=0.15)$.

Conclusions Our results suggest that participants perception of body image correlate well with BMI and can be used in epidemiological studies where availability of objective measures are limited.

\section{SP1-64 A COHORT STUDY ABOUT THE ASSOCIATION BETWEEN METABOLIC SYNDROME AND FTO GENETIC POLYMORPHISM IN A JAPANESE WORKER POPULATION}

doi:10.1136/jech.2011.142976n.41

T Kishimoto, ${ }^{*}$ Y Osaki, A Tahara, M Nagai, T Kawajiri. Division of Environmental and Preventive Medicine, Department of Social Medicine, Faculty of medicine, Tottori University, Yonago, Japan

Introduction The association between the fat-mass and obesityassociated (FTO) genetic polymorphism and the metabolic syndrome (MS) has been suggested by genome-wide association study. We reviewed it using a retrospective cohort study to determine the association between FTO genetic polymorphism and MS in a Japanese worker population.

Methods In the employees of a certain business institution of Shimane Prefecture, subjects were parsons who were without MS at a health examination of 1998 and continued the health examination from 1998 to 2006. We identified three SNPs of the FTO gene of each subject as rs9939609, rs1121980 and rs1558902. For the diagnostic criteria of the MS, we adopted National Cholesterol Education Program-Adult Treatment Panel III (NCEP- ATP III) proposed in 2001. However, we used Body Mass Index 25.0 or more as obese criteria in substitution for abdominal circumference. The number of the subjects was 859 people. The mean observation period was 7.1 years

Results The HR of TA+AA for TT of FT0 genetic polymorphism rs9939609 for the MS incidence adjusted in sex, age, a lifestyle (alcohol consumption, smoking, exercise, satisfaction of the sleep, a diet balance) was 1.48 (95\% CI 1.08 to 2.02); the HR of GA+AA for GG of rs1121980: 1.60 (95\% CI 1.18 to 2.19); the HR of TA+AA for TT of rsl558902: 1.41 (95\% CI 1.04 to 1.92 ).

Conclusion These results suggested that FTO genetic polymorphism may associate the MS incidence by NCEP-ATP III criteria in the Japanese worker population.

\section{SP1-65 PREVALENCE AND INCIDENCE OF CHRONIC CONDITIONS IN AN AUSTRALIAN POPULATION BASED COHORT STUDY: 2000 TO 2010}

doi:10.1136/jech.2011.142976n.42

T Gill, ${ }^{*}$ A Taylor, J Grant, E Dal Grande, R Adams, S Appleton. University of Adelaide, Adelaide, South Australia, Australia

Introduction The North West Adelaide Health Study is a representative population cohort study of approximately 4000 adults aged 18 years and over in the northern and western regions of Adelaide. Cohort assessment has been conducted over a 10-year period to provide baseline and ongoing information about chronic disease and health-related risk factor status from self-reported and biomedically measured data. The study identifies those across the chronic disease continuum from those without disease, those at risk, undiagnosed and diagnosed.

Methods The initial sample was randomly selected and over 4050 participants attended a clinic assessment in Stage 1. The number of participants undertaking a clinic assessment in Stage 2 was $n=3200$ and in Stage 3 approximately $n=2500$. Data have been collected using Computer Assisted Telephone Interview (CATI) surveys, selfcompleted questionnaires and biomedical measurements and include musculoskeletal and respiratory conditions and diabetes and related risk factors.

Results An overview of the study's findings from 2000 to 2010 will be presented, highlighting initial disease prevalence, incidence and characteristics of at-risk populations. This information provides epidemiological evidence relating to relevant conditions and risk factors which can be used in the development of prevention and treatment programs.

Conclusion Chronic diseases play an important role in today's society in terms of costs and impact on the population, both for those with the disease and for the wider community. It is important that these conditions are prevented if possible and managed effectively in order to reduce societal burden.

\section{SP1-66 CHRONIC DISEASE IN SPECIFIC POPULATIONS: AN EPIDEMIOLOGICAL ANALYSIS}

doi:10.1136/jech.2011.142976n.43

${ }^{1} \mathrm{~T}$ Gill, ${ }^{*} \mathrm{~A}$ Taylor, ${ }^{2} \mathrm{~K}$ Price, ${ }^{1} \mathrm{R}$ Pilkington. ${ }^{1}$ University of Adelaide, Adelaide, South Australia, Australia; ${ }^{2}$ University of South Australia, Adelaide, South Australia, Australia

Introduction The rising prevalence of chronic diseases in Australia is a significant challenge to both healthcare systems and the population. While it is the older population that is, the primary focus of chronic disease prevention programs, other population groups are also significantly affected by these conditions and present a challenge to governments and healthcare professionals alike. Factors that influence the development of chronic disease have been comprehensively studied in the older population but other groups have received less focus.

Methods The North West Adelaide Health Study is a cohort study being conducted in the northwest suburbs of Adelaide, Australia. The initial sample was randomly selected and over 4050 participants attended a clinic assessment in Stage 1. Participants undertaking a clinic assessment in Stage 2 was $n=3200$ and Stage 3 approximately $\mathrm{n}=2500$. Data have been collected using Computer Assisted Telephone Interview (CATI) surveys, self-completed questionnaires and biomedical measurements. The chronic conditions examined include asthma, diabetes, cardiovascular disease, chronic obstructive pulmonary disease and osteoporosis.

Results Univariate and multivariate analyses of specific population subgroups such as young people, carers and non-English speaking background demonstrate various associations between the presence of a chronic condition, socioeconomic factors and health service use as well as risk factors such as smoking status, depression and quality of life.

Conclusions Improvements in chronic disease management is becoming a greater imperative in today's society. Targeting of prevention and treatment can occur based on epidemiological analyses. Chronic disease does not discriminate and requires a societal wide perspective. 